\title{
Bücher aus dem Besitz auswärtiger Herrscher
}

Die Württembergische Landesbibliothek Stuttgart verfügt über eine Reihe von Büchern aus dem Vorbesitz regierender Fürsten des deutschen und europäischen Auslands. Die Information über das frühere Besitzverhältnis begegnet dabei in großer Vielfalt der Ausdrucksmittel. Wie die Bände nach Stuttgart kamen, lässt sich manchmal nur vermuten.

Häufig nehmen Kinder auswärtiger Herrscher nach Eheschließung mit Angehörigen des Hauses Württemberg Bücher von persönlicher Wichtigkeit in die neue Heimat mit. Dies gilt z.B. für ein Exemplar der handlichen, 1744 bei Johann Heinrich Meyer in Lemgo verlegten Lutherbibel (B deutsch 1744 03). Ein handschriftlicher Eintrag in vornehmem Französisch auf dem vorderen Vorsatzblatt verrät, dass Dorothea Sophia Friederike von Brandenburg-

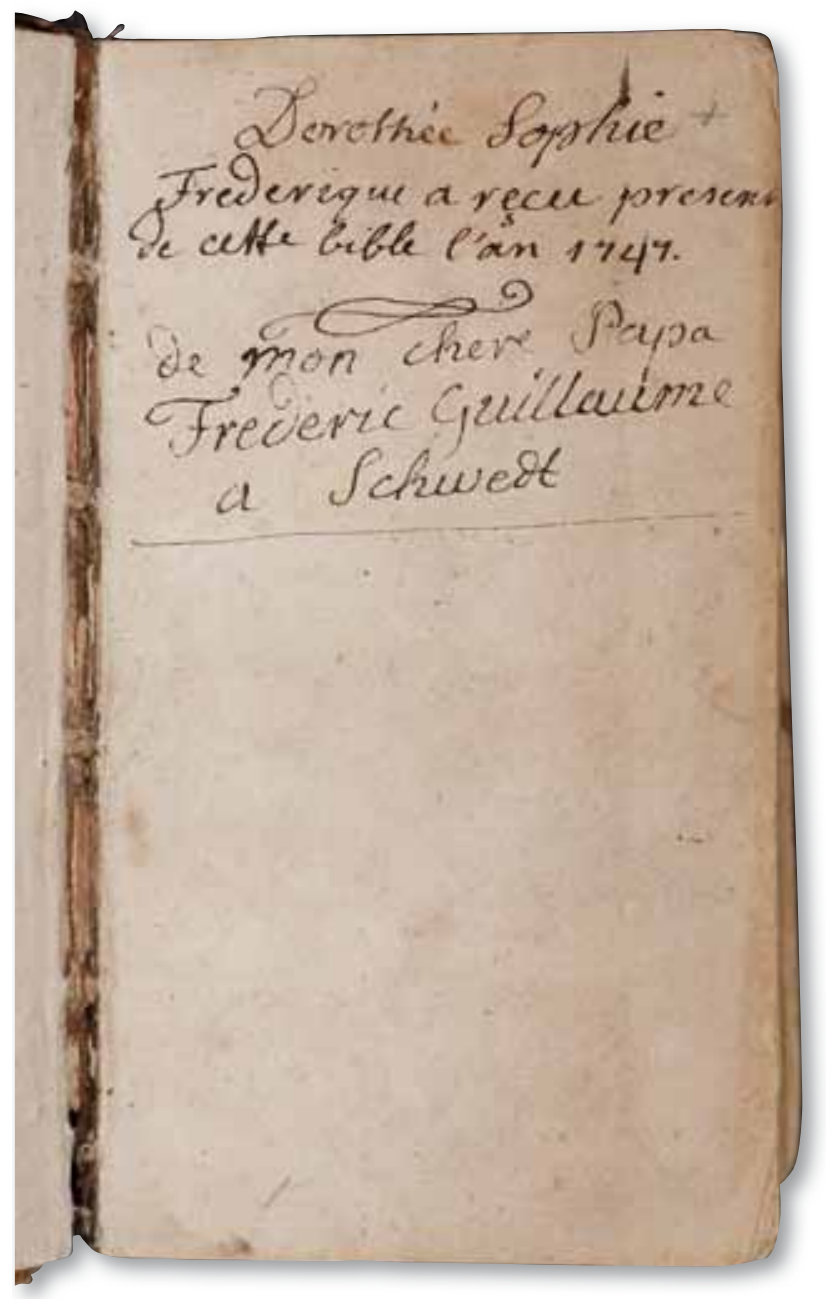

Abb. 1: B deutsch 174402
Schwedt (1736-1798) als Elfjährige diese Bibel von ihrem Vater, Markgraf Friedrich Wilhelm von Brandenburg-Schwedt (1700-1771), als Geschenk erhalten hat: „Dorothée Sophie Frederique a reçu presente cette bible I'an 1747. De mon chere Papa Frederic Guillaume a Schwedt". 1753 heiratet sie den späteren Herzog Friedrich Eugen von Württemberg (1732-1797) und trägt die Daten für Geburt und Taufe ihrer zwölf Kinder auf den hinteren Vorsatzblättern ein.

Vermutlich kommt durch sie auch eine großformatige Bilderbibel mit Kupferstichen von Jan Luyken (1649-1712) (Bg graph. 1700 01) nach Stuttgart. Das ornamentale goldgeprägte Mittelstück wird umsäumt von einem kalligraphisch gestalteten Monogramm mit Krone und Initialen ihrer Großmutter Sophie Dorothea (1687-1757), der Gemahlin des preußischen Soldatenkönigs Friedrich Wilhelm I. (1688-1740). Wohl als Teil der Mitgift zur Hochzeit zwischen

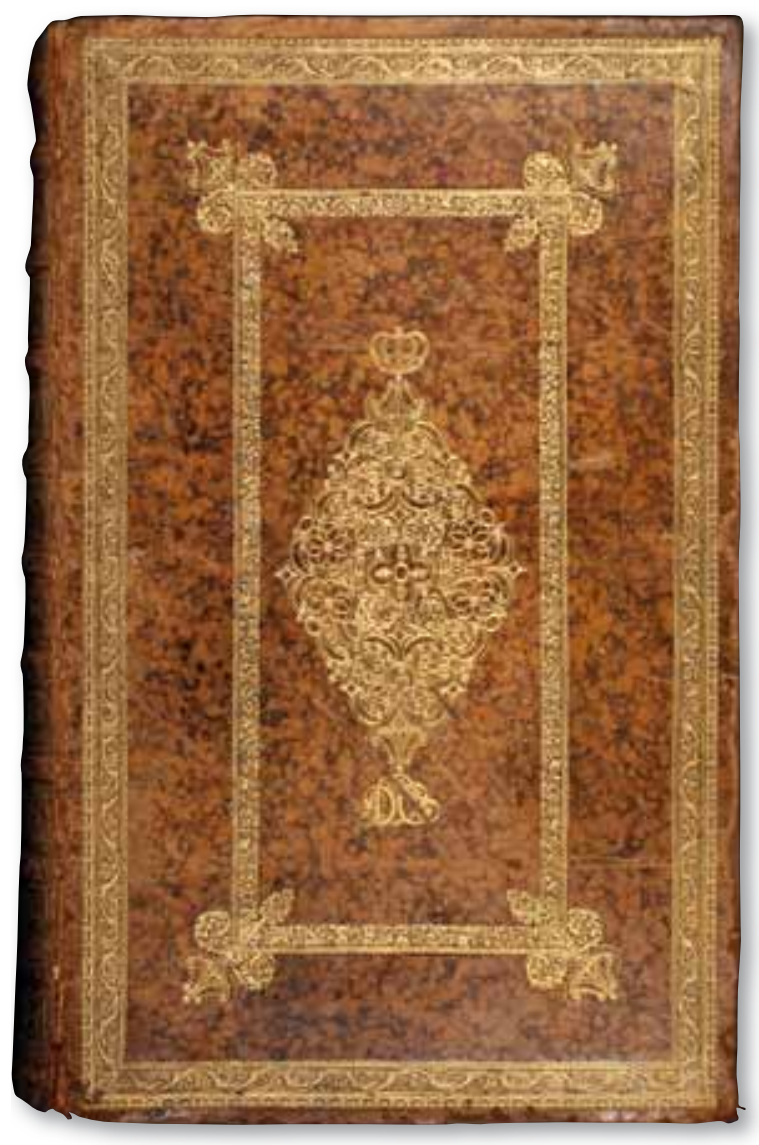

Abb. 2: Bg graph. 170001 
Charlotte Auguste Mathilde (1766-1828) mit dem späteren württembergischen König Friedrich I. (1754-1816) gelangt ein prächtig ausgestattetes Exemplar des anglikanischen Book of Common Prayer, Cambridge 1762 (Theol.fol. 222), nach Stuttgart. Der Band trägt auf Vorder- und Rückdeckel das britische Königswappen Georgs III. (17381820) als goldgeprägtes Supralibros. ${ }^{1}$



Abb. 3: Theol.fol. 222

Heiratspolitik als Mittel der Außenpolitik wird wiederholt ästhetisch manifest in Gestalt sogenannter Allianzwappen-Einbände. Dabei werden die Wappen der beteiligten Territorien durch Plattenprägung als zentrales Verzierungselement des Einbands abgebildet. So findet man auf einem Exemplar des Berichts über die 1575 stattfindende Hochzeit zwischen Herzog Ludwig von Württemberg (1554-1593) und Dorothea Ursula (1559-1583), der Tochter Markgraf Karls II. von Baden-Durlach, das württembergische Wappen auf dem Vorder- und das badische Wappen auf dem Hinterdeckel (R 16 Fri 4).

(1) Von Georg III. stammt vermutlich auch ein reich verziertes Miniaturbuch von 1796 (HBF 2861 / ES 22), bei dem auf dem Schuber das Monogramm GR (= Georg Rex) vermerkt ist.

(2) Katalog der Neuenstädter Bibliothek: Cod.hist.fol. 1072 bzw. Cod. hist.fol. 210.

(3) Löffler, Karl: Geschichte der Württembergischen Landesbibliothek, Leipzig 1923, S. 13.

(4) Vgl. Löffler, Landesbibliothek, S. $20 f$

(5) Dieser Band passt zu den bei einer Versteigerung der Senckenbergischen Stiftsbibliothek 1785 erworbenen Bänden. Dazu Löffler, Landesbibliothek, S. 20.
Komplizierter ist der Weg, den eine von Kaspar Meuser in prächtiger Weise gebundene Bibel von 1564 nach Stuttgart führt (Bb deutsch 156402 / ES IV). Auf dem Einbanddeckel wie auf dem Schnitt wird das sächsische Wappen abgebildet. Das Monogramm F D H Z S steht für „Fräulein Dorothea Herzogin zu Sachsen“ (1563-1587), d.h. die Tochter des Kurfürsten August von Sachsen (1526-1586) und seit 1585 Ehefrau des späteren Herzogs Heinrich Julius von Braunschweig-Wolfenbüttel (15641613). Ihrer Enkeltochter gleichen Namens (16071634), seit 1623 vermählt mit dem bibliophilen Herzog August II. von Braunschweig-Wolfenbüttel (1579-1666), fiel das Buch laut handschriftlicher Eintragung bei einer Erbteilung 1622 zu. Deren Tochter Clara Augusta (1632-1700) heiratet 1653 Herzog Friedrich von Württemberg-Neuenstadt (1615-1682), dessen große Privatbibliothek 1688 nach Stuttgart verkauft und mit der Bibliothek des Oberrats vereinigt wird. ${ }^{2} 1776$ werden die Bücher des Regierungsrats (früher: Oberrat) in die Herzogliche Öffentliche Bibliothek inkorporiert. ${ }^{3}$ Es liegt nahe, dass die Prachtbibel über mehrere Generationen und über dynastische Grenzen hinweg jeweils über die weiblichen Angehörigen der Fürstenhäuser weitergegeben wird und schließlich nach Stuttgart gelangt.

Andere Bände werden dem für seine Bibliophilie bekannten württembergischen Herzog Carl Eugen von Institutionen oder anderen Herrschern geschenkt, sei es um ihn für weitere Geschäftsbeziehungen geneigt zu machen, sei es als Mittel zwischenstaatlicher Diplomatie. Daneben sind zahlreiche Einkäufer im Auftrag Carl Eugens in Europa unterwegs. ${ }^{4}$ Das gilt z.B. für eine Wittenberger Lutherbibel von 1572 (Bb deutsch 1572 02), die sich laut handschriftlicher Eintragung zunächst im Besitz Gräfin Katharinas von Ostfriesland (15391610), der Tochter des Schwedenkönigs Gustav Wasa (1496-1560) befindet. Wie Exlibris und weitere Eintragungen verraten, gelangt die Bibel später in den Besitz des von pietistischer Frömmigkeit geprägten Frankfurter Arztes Johann Christian Senckenberg (1707-1772) bzw. des Collegium Medicum als Haupterben, das sie 1785 an Carl Eugen verschenkt. $^{5}$ 
Ein 1776 in Rom gedruckter Band über katholische Glaubensinhalte (HBFa 831) wird als Schenkungsexemplar der Römischen Kurie in italienischer Manier, jedoch mit württembergischem Wappen gebunden. Für die katholische Theologie ist die Anknüpfung der Gnade an das Natürlich-Kreative bzw. Schöne zentral. Bibliophil gestaltete Einbände erhalten als Ausdruck der Offenheit für Sinnenfreude die Funktion konfessioneller Apologetik. So kann man davon ausgehen, dass auch die beiden Einbände aus dem Vorbesitz der Päpste Klemens VII. (Pontifikat 1667-1669) (HBFb 200 / ES VI) bzw. Klemens XI. (Pontifikat 1700-1721) (Phil.oct. 7535) von päpstlichen Gesandten dem katholischen Herzog übergeben werden. ${ }^{6}$

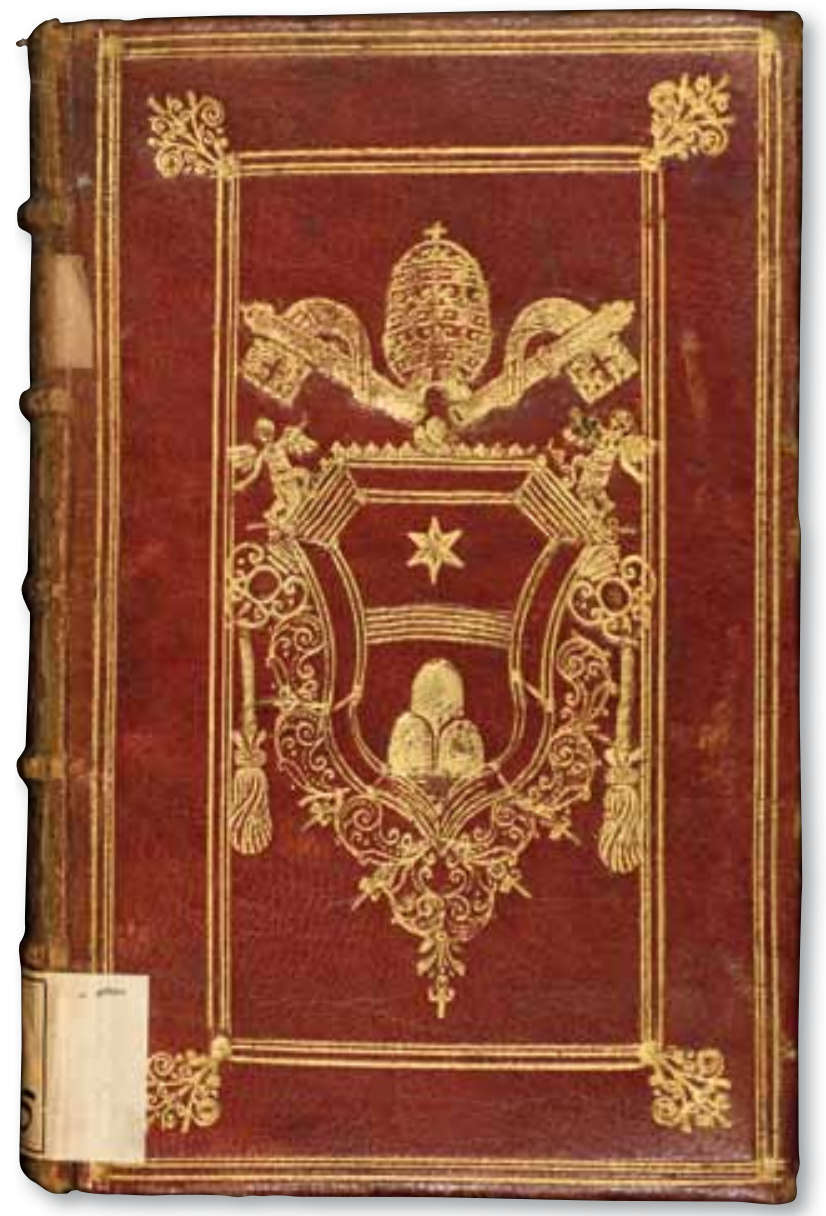

Abb. 4: Phil.oct. 7535

Vermutlich als Geschenk des österreichischen Hofes an Herzog Carl Eugen kommt ein Band mit dem Supralibros des römisch-deutschen Kaisers Karl VI. (1685-1740) in die Stuttgarter Bibliothek (Phil.qt. 122). Ähnliches könnte für eine schwedische Bibel von 1715 mit dem Monogramm König Karls XII. (1682-1718) gelten (B schwed. 1715 01). ${ }^{7}$ Indem man Bücher mit Besitzkennzeichen bereits verstorbener Herrscher schenkt, kann man Herzog Carl Eugen günstig stimmen, ohne die von den jeweils amtierenden Fürsten erworbenen Bände antasten zu müssen.

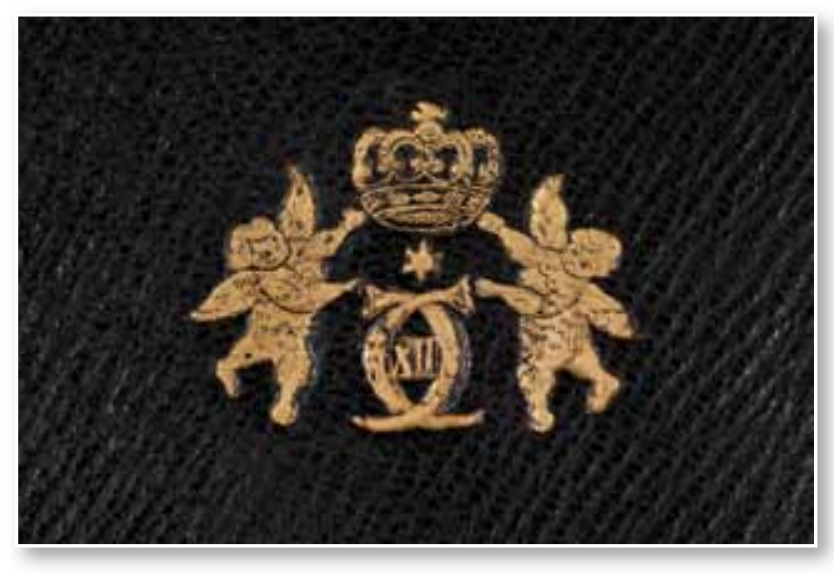

Abb. 5: B schwed. 171501

Der Gang des Widmungsexemplars für einen weiteren Vertreter des Hauses Habsburg, nämlich Erzherzog Maximilian (1558-1618), ist klarer (HBb 1247-1 / ES X). Er ist ab 1590 Hochmeister des Deutschen Ordens und vermacht testamentarisch seine umfangreiche Privatbibliothek diesem Orden. Aufgrund von Erbstreitigkeiten gelangt ein Großteil der Bände erst 1627 in die Bibliothek der Deutschordenskommende Mergentheim, worauf beim vorliegenden Band das Brandkreuz auf dem Schnitt hinweist. Nach der Säkularisation der Klosterbibliotheken ab 1803 gelangen die Bände schließlich in die Handbibliothek bzw. Öffentliche Bibliothek in Stuttgart sowie in die Universitätsbibliothek Tübingen. ${ }^{8}$

Einige Bibeln aus dem Besitz auswärtiger Herrscher kommen als Teil der 1784 erworbenen Sammlung des Kopenhagener Pastors Josias Lorck in die Stuttgarter Bibliothek. Dazu gehört ein venezianischer Vulgata-Druck von 1609 aus der Privatbibliothek des englischen Königs Jakob I. (15661625) (Bb lat. 1609 02). Auch kleinformatige Bibeln mit blind- oder goldgeprägten Monogrammen

(6) Zu Buchgeschenken als Mittel der Diplomatie: Löffler, Landesbibliothek, S. 19, $21 \mathrm{ff}, 36$.

(7) Denkbar wäre aber auch, dass es sich um einen Band aus der 1785 teilweise gekauften Aurivillischen Bibliothek in Stockholm handelt. Dazu Löffler, Landesbibliothek, S. 21.

(8) Vgl. Stampfer, Ursula: Die Hofbibliothek Erzherzog Maximilians III. von Österreich (1558-1618), Diss. Innsbruck 2008, S. 62f, $70 f$. Stampfer identifiziert zudem 62 weitere Drucke im Bestand der WLB Stuttgart mit dieser Provenienz, dazu vier Drucke mit großer Wahrscheinlichkeit, außerdem zwölf Handschriften (ebd., S. 517-520). Ausgangspunkt ist dabei ein handschriftlicher Katalog der Bibliothek Maximilians von 1619, deren Titel mit dem Bestand der Empfängerbibliotheken abgeglichen werden. Hinzu kommen eindeutige Eigentumsvermerke bzw. Signaturen aus der Bibliothek Maximilians. 
König Ludwigs XIII. von Frankreich (1601-1643) (B polygl. 1629 01) sowie der dänischen Königin Sofie Magdalene (1700-1770) (B dän. 1728 01; B dän. 173801 ) stammen aus dieser Quelle. ${ }^{9}$ Besonders ansprechend ist eine Bibel mit teilweise aus Silberfäden gesticktem Gewebeeinband, der mit Motivik (Elch) und Farbgebung (blau, gelb) auf die Heimat der Vorbesitzerin Königin Christine von Schweden (1626-1689) verweist. Lorck teilt mit, dass es sich um die persönliche Handbibel der dann zum Katholizismus konvertierten Königin gehandelt haben soll (B schwed. 163301 / ES 5). Auch Bücher aus

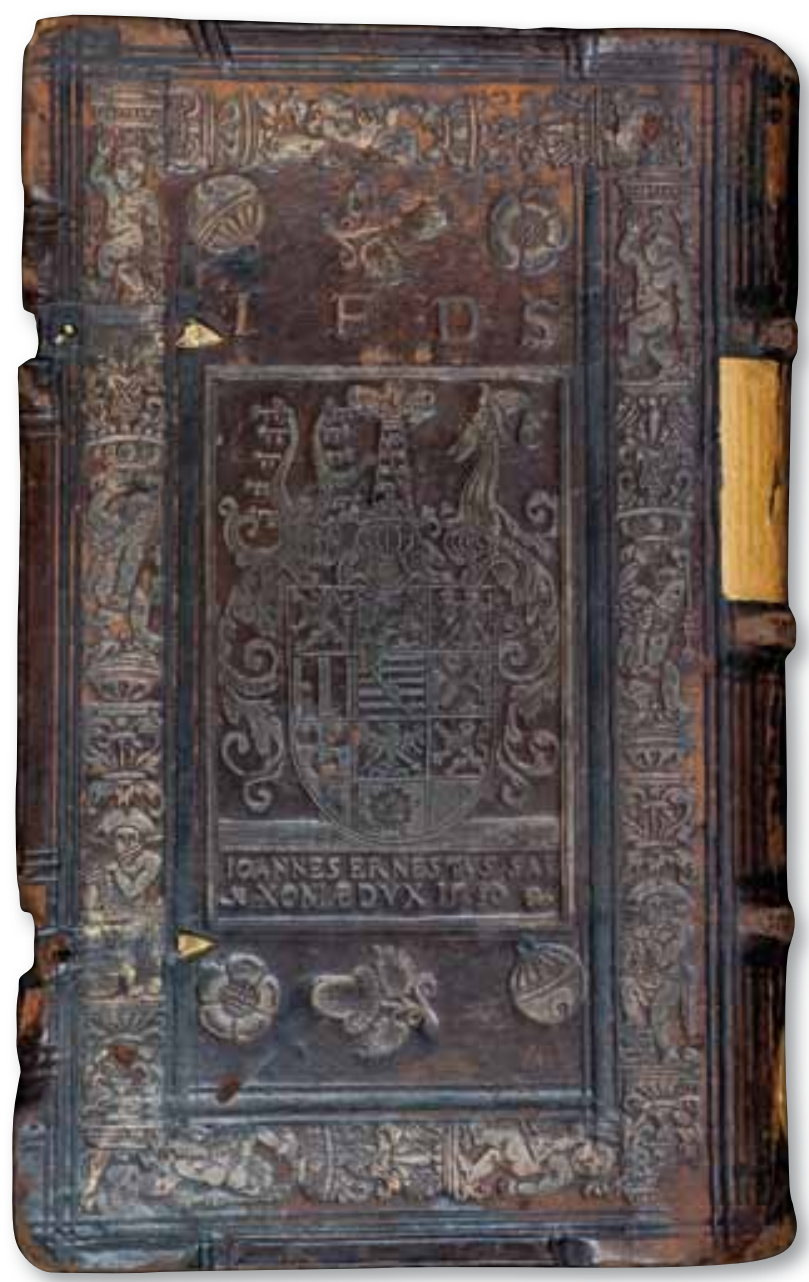

Abb. 6: B lat. 153705

(9) Eine weitere dänische Bibel (B dän. 1740 03) weist das Monogramm Sofie Magdalenes auf, allerdings ohne Besitzvermerk Lorcks.

(10) Außerdem kommt eine von Herzog August II. von BraunschweigWolfenbüttel (1579-1666) 1665 an Johann Saubert d.J. (1638-1688) gestiftete Bibel durch die Sammlung Lorck in die Landesbibliothek (B deutsch 1531 01).

(11) Catalogue des livres imprimés et manuscrits de la bibliothèque de feu Monseigneur Le Prince de Soubise, Maréchal de France. Dont la vente sera indiquée par affiches au mois de janvier 1789, Paris 1788.

(12) Dazu Löffler, Landesbibliothek, S. 30. Carl Eugen, Tagbücher seiner Rayßen nach Prag und Dresden, durch die Schweiz und deren Gebürge, nach Nieder Sachßen und Dännemarck, durch die angesehensten Clöster Schwabens, auf die Franckforter Messe, nach Mömpelgardt, nach den beiden Königreichen Franckreich und Engelland, nach Holland und manch anderen Orten. In den Jahren 1783-1791, hrsg. von Robert Uhland, Tübingen 1968, S. 291-304.

(13) Von Ludwig XV. stammt zudem Franz.G.qt. 118. Von Ludwig XIV. befinden sich Kirch.G.fol. 181-1 bis -37 sowie Allg.G.fol. 684 und HBF 2864 im Bestand. dem Besitz weniger bedeutender Fürsten wie Herzog Johann Ernst von Sachsen-Coburg (15211553) sind Teil der Lorckschen Bibelsammlung. In diesem Fall (B lat. 1537 05) wird auf dem Rückdeckel mit einer Platte das Landeswappen mit Beischrift „IOANNES ERNESTVS SAXONIAE DVX 1550 blindgeprägt abgebildet. ${ }^{10}$

Herzog Carl Eugen beteiligt sich 1789 an der Auktion der Privatbibliothek des Generals Prinz Charles de Rohan Soubise (1715-1787). Die meisten der dort erworbenen Bände zeichnen sich durch prächtige Wappeneinbände aus. Auch das Supralibros der französischen Könige Ludwig XIV. (1638-1715)

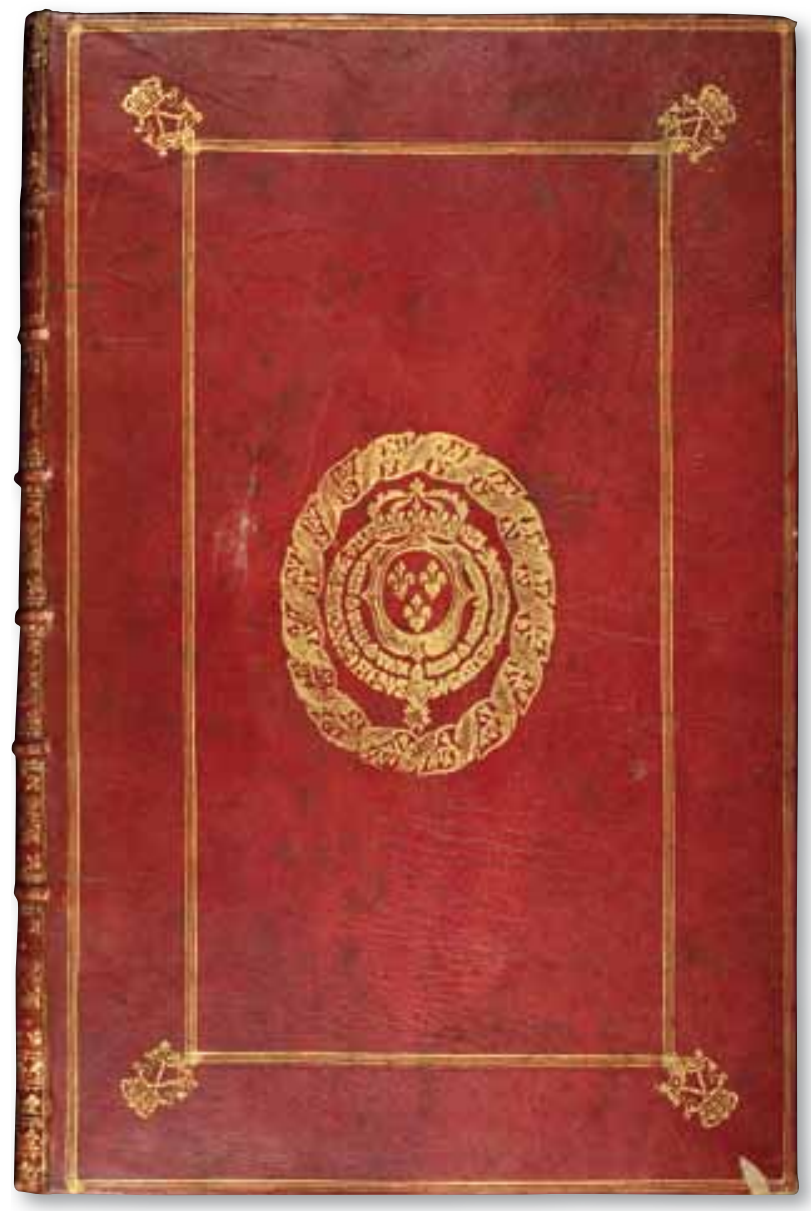

Abb. 7: Sch.K.fol. 44

(HBF 2867 / ES 46) und Ludwig XV. (1710-1774) (Theol.fol. 482) ist zu finden. Weitere durch ein goldgeprägtes Wappen-Supralibros diesen Königen zuzuordnenden Bände werden im Katalog der Soubise-Sammlung nicht aufgeführt. ${ }^{11}$ Es ist davon auszugehen, dass Herzog Carl Eugen sie bei seiner Reise nach Paris vom 1. bis 22.2.1787 erworben hat. ${ }^{12}$ Inhaltlich wichtig ist dabei vor allem ein 1743 gedruckter Katalog der Privatbibliothek Ludwigs XV. (Sch.K.fol. 44). ${ }^{13}$ 
Bei seiner Reise nach Dänemark 1784 hat Herzog Carl Eugen vermutlich auch die großformatigen Bände über die dänische Flora und Fauna aus dem Besitz des damals amtierenden Königs Christian VII. (1749-1808) als Geschenk erhalten (Nat.G.fol. 147-1 bis -5; Nat.G.fol. 804). Die prächtige äußere Dekoration mit Wappen-Supralibros passt zu den aufwendigen Illustrationen im Inneren. ${ }^{14}$

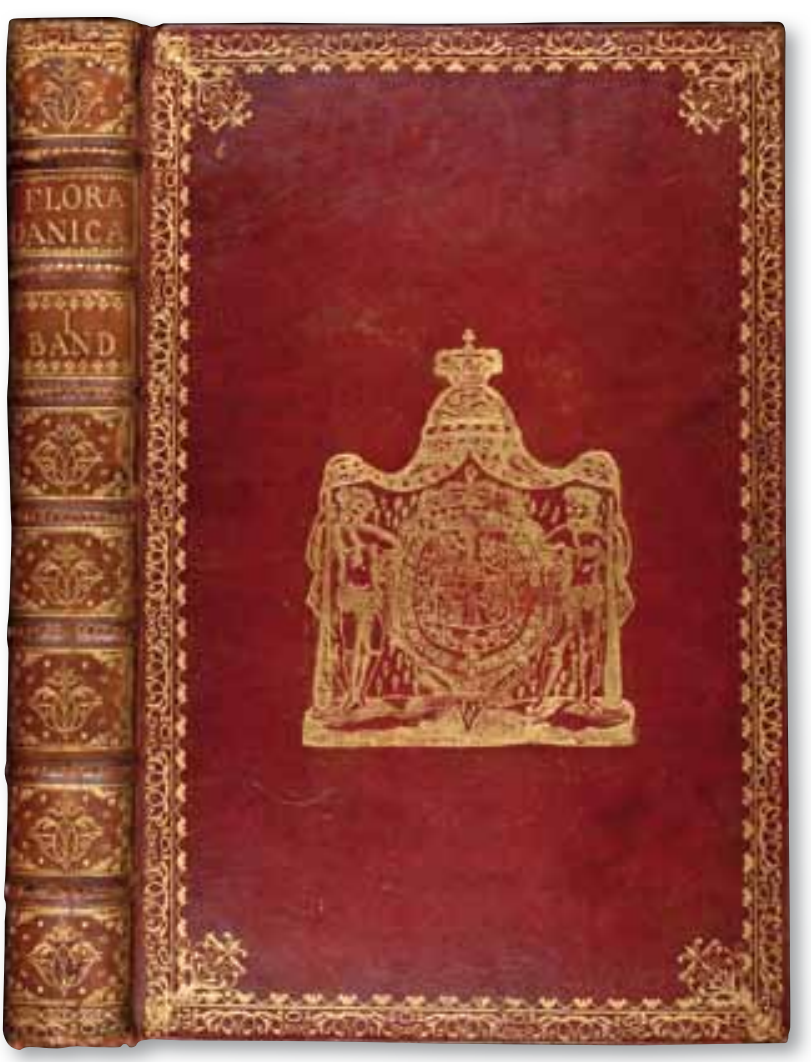

Abb. 8: Nat.G.fol. 147-1

Seltener gelangen Bücher fürstlicher Provenienz in der Zeit nach Herzog Carl Eugen in die spätere Württembergische Landesbibliothek. Dabei handelt es sich wahrscheinlich um antiquarische Einzelerwerbungen oder Schenkungen. Das gilt für ein 1820 gedrucktes Lehrbuch über die Arktis aus dem Besitz König Friedrichs VI. von Dänemark (1768-1839). Ein bescheidenes Monogramm auf dem Buchrücken deutet den früheren Besitzer an (Geogr.oct. 6569-1 bis -2). Der französische Bürgerkönig Louis Philippe (1773-1850) ist im Bestand der Landesbibliothek Stuttgart mit zwei Bänden vertreten, die durch ein goldgeprägtes Monogramm bzw. Wappen auf Buchdeckeln bzw. Rücken eindeutig ihrem Vorbesitzer zugeordnet werden können (Sch.K.oct. 2763; Franz.G.qt. 242). Über den früheren Direktor der Württembergischen Landesbibliothek, Wilhelm Hoffmann d.J.



Abb. 9: Sch.K.oct. 2763

(1901-1986) gelangte eine englische Bibel von 1836 (Ba engl. 1836 02) in den Bestand, die im Jahr 1860 seinem Großvater, dem Berliner Generalsuperintendent Wilhelm Hoffmann d.Ä. (18061873), von Kronprinz Friedrich Wilhelm von Preußen, dem späteren deutschen Kaiser Friedrich III. (1831-1888) und seiner Frau Prinzessin Viktoria (1840-1901) - Tochter der englischen Königin Victoria (1819-1901) - gewidmet wird.

In der Tatsache der Weitergabe und Pflege von Büchern über Generationen und Territorien hinweg kommt deren besondere Wertschätzung zum Ausdruck.

\section{Christian Herrmann}

(14) Der erst 1792 erschienene Band 6 von Nat.G.fol. 147 und die folgenden Bände sind anders gestaltet. Auch das spricht für diesen Erwerbungsweg und -zeitpunkt. Es ist bekannt, dass auch eine in Seide gebundene dänische Handschrift (Cod.iur.fol. 109) von Christian VII. dem württembergischen Herzog bei seinem Aufenthalt in Kopenhagen geschenkt wurde. Vielleicht wurde Carl Eugen bei der Besichtigung der königlichen Handbibliothek auf Schloss Fredensborg am 17.2.1784 auf die Bände aufmerksam, die man ihm dann geschenkt hat (dazu Carl Eugen, Tagbücher, S. 160). 